Article

\title{
Shape Optimum Design by Basis Vector Method Considering Partial Shape Dependence
}

\author{
Qiong $\mathrm{Wu}^{1, *}$, Hairui Zhang ${ }^{1}$, Wei Zhao ${ }^{2}$ and Xilu Zhao ${ }^{3}$ \\ 1 College of mechanical and Electronic Engineering, Nanjing Forestry University, Nanjing 210037, China; \\ zhanghairui@njfu.edu.cn \\ 2 Weichai Global Axis Technology Co. Ltd., Minami-aoyama Minato Tokyo, Tokyo 107-0062, Japan; \\ wdgazhaowei@weichaigbaxis.com \\ 3 Saitama Institute of Technology, Fukaya 369-0293, Japan; zhaoxilu@sit.ac.jp \\ * Correspondence: wuqiong@njfu.edu.cn
}

Received: 5 October 2020; Accepted: 1 November 2020; Published: 5 November 2020

\begin{abstract}
Regarding the case of complicated structural shape optimization, there are cases where there are partial shapes such as holes and irregularities inside the structure. Concerning the complex structural optimization shape, the relationship between the external boundary shape and the internal local shape should be maintained, and how to change the internal partial shape while maintaining a subordinate relationship with the external form has become an important issue. Currently, there is no good solution to this kind of problem using general optimization design software. Therefore, this paper proposes to use the basic vector method to solve the local shape dependency problem of partial shapes. First, this paper classifies the subordinate problems of partial shape into three primary patterns, theoretically proving a method for controlling subordinate relationships of partial forms, respectively. Then, the research also provides two classical application examples: shape optimization of a steam turbine implantation section and stress distribution optimization of an engine mount bracket. The results show that the optimization method is effective for the partial shape subordination problem in complex structural shape optimization problems. Finally, the study examines the problem of making a vectorial vector, a correlation between the basis vector and the remeshing problem of the analysis model in shape optimization, and further substantiates the validity of the method proposed by the body using the analysis result of the actual structural shape optimization case.
\end{abstract}

Keywords: structural shape optimization; basis vector method; partial shape dependence; remeshing problem

\section{Introduction}

To improve the quality of machine products, applying topological optimization technology to actual product structure design has received much attention. Since the 1980s, in the industrial world such as aerospace and automobiles, the structural analysis technique using the finite element method and a mathematical programming method had been the norm [1]. Research on structural optimization for obtaining the optimum structural composition has been actively conducted after satisfying restrictive conditions such as strength and rigidity [2].

Since the beginning of the 2000s, researchers have improved the analysis technology using supercomputers and other hardware, collision analysis and noise analysis, along with the approximate analytical model, have introduced the experimental design method and the neural network, and the range of study of optimization and the scale of analysis model are further expanded.

Recently, with the progress of analytical technology, the need for structural topological optimization has been increasing more and more from the field of design development. Regarding conventional 
structural shape optimization, depending on the type of design variable, it can be classified into size optimization, phase optimization, and shape optimization.

During size optimization, the most general setting should consist of a determination for every point in space, whether there is material at that point or not, such Azegami, H. proposed as a solution to shape optimization problems of domain boundaries in which boundary value problems of partial differential equations are defined [3]. Alternatively, for a FEM (Finite element method) discretization, every element is a potential void or structural member. Since the design variables of dimension optimization are simple, it is currently the situation that there are relatively few cases that can be applied to complicated structural topological optimization problems.

During topological optimization, the element types and element densities of the analysis model are used as design variables, and the number of elements increases or decreases during the optimization analysis [4]. He, D., et al., mentions only the critical substructure to which the internal force of the structure is transmitted remains, when the so-called optimum phase structure can be obtained. However, the shape of the optimum structure obtained by phase optimization is difficult to predict in advance, it is difficult to use at the stage of detailed design, and there are features to be applied to the initial design $[5,6]$.

During topological optimization, by changing the boundary shape of the structure using a parameter of a combination of appropriate interpolation functions, and by directly changing the design variable via the design variable, the design variable is changed in the middle of the optimization analysis, therefore, it is possible to control the shape change [7]. Lu, J., et al., find it is common to use the definition and numerical formulation of CAD (computer-aided design) shape data to parameterize the structural shape change [8]. However, similar to dimension optimization, there is a remaking problem that appropriately adjusts the analysis mesh inside the structure under the shape change of the structure boundary.

Conversely, the researchers proposed a topological optimization method based on the reverse variation principle on the edge, since it is a method for finding the optimum boundary shape mathematically to realize the design target under the given load condition and constraint condition $[9,10]$. Although it is applied to structural design or independent part design, it is easy to see that it is difficult to apply to complicated topological optimization problems often found in the design site [11].

Observations from earlier studies [12] during the actual machine part topological optimization problem find, as the external boundary shape of the structure is changed, the internal shape of the hole or the uneven partial shape is always adjusted according to requirements such as the assembly relationship and the processing condition between the part and the part. It is common to have a certain partial shape dependency. Alternatively, it is required that a partial shape dependency relationship always exists between the boundary shape outside the structure and the internal partial shape in the course of shape optimization $[13,14]$.

To solve this partial shape subordination problem, some researchers have applied the basis vector method based on the basic shape change pattern [15,16], like Tang, Y., and Mam, K., et al., who proposed a COBYLA optimization method, and Wang, C., et al., who proposed evaluation of manufacturing constraints from the early stages of the conceptual design to perform an optimization coherent with the manufacturing technology chosen. Actually, when optimizing the structure using the basis vector method, one first considers the structural processing condition of the design object and the assembling relation with other parts, from the designer $[17,18]$, then some basic shape change patterns and their change range. Then, each new basic shape change pattern is multiplied by a weighting coefficient and added to form a new structure shape. Each weighting coefficient is used as a design variable as it is, thereby obtaining an optimal weighting factor $[19,20]$. Here, since each basic shape change pattern is expressed in the form of a node coordinate vector having a similar phase configuration, this method is called a basis vector method. To the authors' knowledge, there are no studies of shape optimization considering the partial shape dependence problem. However, when the basic vector method is applied to complex structural, topological optimization problems, there is no theoretical 
research on the partial shape dependency problem, and the basic vector problems which depend on the relationship of multiple basic shape change patterns are not solved [21]. This has a significant impact on the solution of complex structure topological optimization problems. While these simple approaches are adequate for many applications, it is nevertheless clear for practitioners that they can become insufficient to address the partial shape dependency optimization method [22,23].

Conversely, many researchers have been making efforts to add optimum design functions in general-purpose analysis codes such as ANSYS and HyperWorks but, due to the complexity and difficulty of partial shape subordination problems, many cases have stopped halfway.

Until now, the partial shape dependency problem in complicated structural shape optimization has not been solved very well, and there is no theoretical research on the partial shape dependency problem. Here, to solve the complicated structural shape optimization problems, some important issues are handled and applied in detail when applying the basis vector method.

First, considering the partial shape dependence problem and the basis vector method, the subordinate relationship of the partial shape inside the structure is divided into three basic cases with the change of the external shape of the structure. The correctness of the basic vector method is verified using mathematical theory, which provides a theoretical basis for the basic vector method used in complex shape optimization design. Additionally, the basic vector generation and mesh regeneration problems in the practical application of the basic vector method are discussed, and the corresponding algorithms are given. The basic vector method proposed in this paper is used to analyze the complex shape optimization design of a two-dimensional turbine blade and a three-dimensional engine base, and the optimization design results verify the local shape dependence problem.

\section{Partial Shape Subordination Problem}

Here, it will be described using a simple example shown in Figure 1. The initial shape is shown in Figure 1a, where we let the design variable $x$ be the width of the rectangular plate, the diameter of the hole in the center is $d_{0}$, the distance from the hole to the left boundary is $a_{0}$, and the distance to the right boundary is $b_{0}$. Shown in Figure 1, when changing the design variable $x$ to $x+\Delta x$, the shape of the holes inside is considered to be four change patterns from Case1 to Case4.

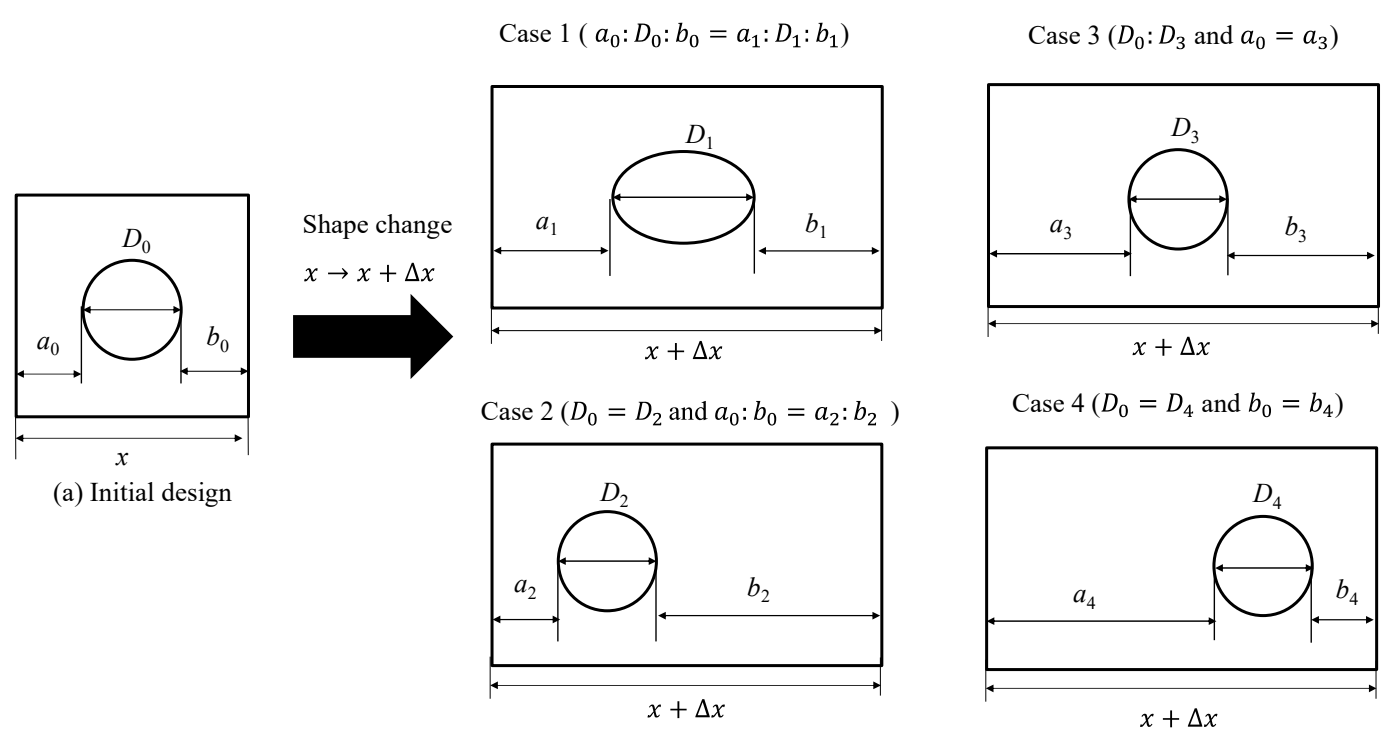

(b) Final design

Figure 1. Control of inside form in the topological optimization problem. (a) Initial design, (b) Final design. 
Case1: The ratio of the lateral length of the hole to the side length is made constant, and the hole changes from a circle to an ellipse.

$$
a_{0}: D_{0}: b_{0}=a_{1}: D_{1}: b_{1}
$$

Case2: Positions of holes which are always fixed regardless of changes in the design variables; the holes are fixed in the center.

$$
D_{0}=D_{2} \text { and } a_{0}: b_{0}=a_{2}: b_{2}
$$

Case3: The distance from the hole to the left boundary is made constant, and the hole and the left boundary are fixed.

$$
D_{0}=D_{3} \text { and } a_{0}=a_{3}
$$

Case4: The distance from the hole and the hole to the right boundary is made constant, and the hole and the right boundary are fixed.

$$
D_{0}=D_{4} \text { and } b_{0}=b_{4}
$$

According to the change of shape design variables described above, there are many subordinate problems during the partial shapes change; this problem actually exists in the shape optimization problem of machine parts, which has a great influence on practical use.

\section{Basis Vector Method Used for Shape Optimization}

To deal with the partial shape dependency problem mentioned in the previous section, the basis vector method is applied. The basis vector method is a method in which a movement of a certain group of nodes is regarded as a pattern of one shape change, corresponding with one shape design variable for some shape change patterns and adds each weighting factor to obtain a new shape.

Here, the principle of the basis vector method will be explained using the example shown in Figure 2. Figure $2 a$ shows the initial shape, Figure $2 b$ shows the basic shape change pattern 1 in which the entire shape falls forward. Figure 2 c shows the basic shape change pattern 2 , in which the upper-end face of the shape is reduced by half in the front-rear direction. Figure $2 \mathrm{~d}$ shows the basic shape change pattern 3 in which the upper-end face of the shape is reduced by half in the lateral direction. However, the mesh structure (element connectivity) of the four analysis models shown in Figure 2 are the same, and only the node coordinate values are different. Here, the node coordinate values of the four analysis models are arranged in the same order, represented by vectors $\overrightarrow{\alpha_{0}}, \overrightarrow{\alpha_{1}}, \overrightarrow{\alpha_{2}}, \overrightarrow{\alpha_{3}}$ respectively, $\overrightarrow{\alpha_{0}}$ is referred to as an original vector while $\overrightarrow{\alpha_{1}}, \overrightarrow{\alpha_{2}}$ and $\overrightarrow{\alpha_{3}}$ are called basis vectors.

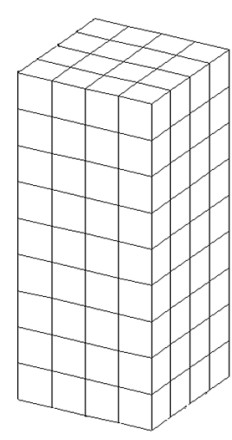

(a)

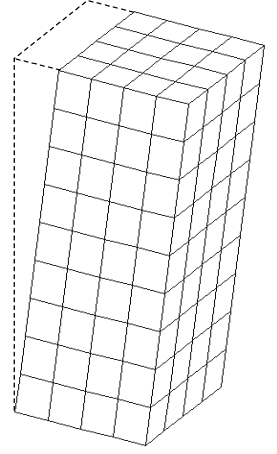

(b)

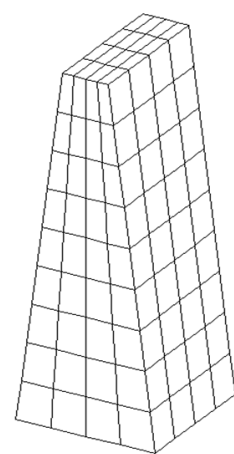

(c)

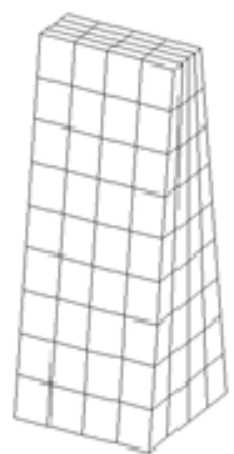

(d)

Figure 2. An example of original and basis shapes. (a) Original shape, (b) Basis Shape 1, (c) Basis Shape 2, (d) Basis Shape 3. 
Further, each coordinate vector $\overrightarrow{\alpha_{0}}, \overrightarrow{\alpha_{1}}, \overrightarrow{\alpha_{2}}, \overrightarrow{\alpha_{3}}$ is multiplied by a weighting coefficient $x_{0}, x_{1}, x_{2}$, $x_{3}$ respectively. Adding the results, the linear combination creates a new node coordinate vector as follows:

$$
\vec{\alpha}=x_{0} \overrightarrow{\alpha_{0}}+x_{1} \overrightarrow{\alpha_{1}}+x_{2} \overrightarrow{\alpha_{2}}+x_{3} \overrightarrow{\alpha_{3}}
$$

When applying Equation (5) to the topological optimization problem in practice, the original vector $\overrightarrow{\alpha_{0}}$ and the basis vectors $\overrightarrow{\alpha_{1}}, \overrightarrow{\alpha_{2}}, \overrightarrow{\alpha_{3}}$ do not always change while the reference data of shapes change at all times. By changing the design variable $x_{0}, x_{1}, x_{2}, x_{3}$, the corresponding node coordinates of the new shape can be calculated directly using Equation (5).

However, when giving the same value to the original vector $\overrightarrow{\alpha_{0}}$ and the basis vectors $\overrightarrow{\alpha_{1}}, \overrightarrow{\alpha_{2}}, \overrightarrow{\alpha_{3}}$, this paper considers the geometric requirement that the new node coordinate vector obtained by Equation (5) becomes the same. The node coordinates of the new shape corresponding to that can be calculated directly using Equation (5):

$$
x_{0}+x_{1}+x_{2}+x_{3}=1
$$

Substituting Equation (6) into Equation (5), the coordinate calculation formula of the basis vector method used for shape optimization is obtained as follows:

$$
\vec{\alpha}=\overrightarrow{\alpha_{0}}+x_{1}\left(\overrightarrow{\alpha_{1}}-\overrightarrow{\alpha_{0}}\right)+x_{2}\left(\overrightarrow{\alpha_{2}}-\overrightarrow{\alpha_{0}}\right)+x_{3}\left(\overrightarrow{\alpha_{3}}-\overrightarrow{\alpha_{0}}\right)
$$

Substituting $x_{1}=0, x_{2}=0.5, x_{3}=0.5$ into Equation (7), the analysis model shown in Figure 3 can be formed using the obtained coordinate vector. Comparing Figures 1 and 3 , the shape obtained by combining the base shape 1 with $0 \%$, the basis shape 2 at $50 \%$ and the basis shape 3 at $50 \%$, according to the value of the design variable, is shown in Figure 3. Therefore, using the design variables $x_{1}, x_{2}$, $x_{3}$, it may be said to be the adoption rate of each basis shape.

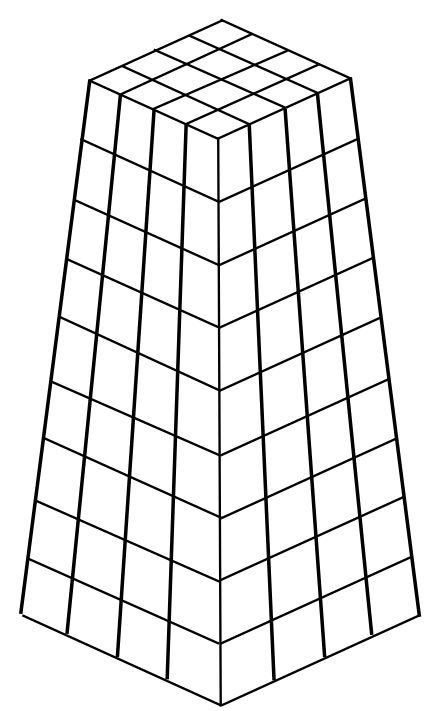

Figure 3. The new basis shape with a leaner relationship.

Regarding this, since the design variable $x_{1}, x_{2}, x_{3}$ is a continuous variable, it is conceivable that the number of shapes formed by calculation using Equation (7) is infinite. Furthermore, the coordinate calculation of the basis vector method expressed by Equation (7) can be generalized when there are several basis vectors.

$$
\vec{\alpha}=\overrightarrow{\alpha_{0}}+x_{1}\left(\overrightarrow{\alpha_{1}}-\overrightarrow{\alpha_{0}}\right)+x_{2}\left(\overrightarrow{\alpha_{2}}-\overrightarrow{\alpha_{0}}\right)+\ldots \ldots+x_{n}\left(\overrightarrow{\alpha_{n}}-\overrightarrow{\alpha_{0}}\right)
$$


However, it is an important research subject, whether it is possible to solve the partial shape subordination problem, as shown in Figure 1 by using Equation (8).

\section{Basis Vector Method and Partial Shape Dependency Relation}

Analyzing the partial shape dependency relationship shown in Figure 1, it can be summarized into the following three basic fundamental relationships:

(1) The corresponding relation of a line segment is composed of any two points.

(2) There is a proportional relationship of distance between three arbitrary points.

(3) The relationship of three arbitrary points is maintained.

Thus, if these three basic subordinations are set, the complex partial shape subordination formed by them also can be ensured.

\subsection{Corresponding Relation of a Line Segment Composed of Any Two Points}

Considering two arbitrary points $a$ and $b$ inside the structure, and taking direction vectors from point $a$ to point $b$ in the original shape, the basic shape and transformed new shape are as follows:

$$
\begin{aligned}
\overrightarrow{a_{0} b_{0}} & =\left\{x_{b 0}-x_{a 0}, y_{b 0}-y_{a 0}\right\}^{T} \\
\overrightarrow{a_{1} b_{1}} & =\left\{x_{b 1}-x_{a 1}, y_{b 1}-y_{a 1}\right\}^{T} \\
\overrightarrow{a b} & =\left\{x_{b}-x_{a}, y_{b}-y_{a}\right\}^{T}
\end{aligned}
$$

Considering if the shape transformation on the weighting factor is $\omega_{0}$ and $\omega_{1}$, the direction vector after transformation according to Equation (8) is represented by Equation (12),

$$
\overrightarrow{a b}=\left\{\left(w_{0} x_{b 0}+w_{1} x_{b 1}\right)-\left(w_{0} x_{a 0}+w_{1} x_{a 1}\right),\left(w_{0} y_{b 0}+w_{1} y_{b 1}\right)-\left(w_{0} y_{a 0}+w_{1} y_{a 1}\right)\right\}^{T}
$$

Taking Equations (9) and (10) into consideration, the above equation becomes Equation (13)

$$
\overrightarrow{a b}=w_{0} \vec{a}_{0} \vec{b}_{0}+w_{1} \vec{a}_{1} \vec{b}_{1}
$$

Considering Equation (13), it is found that the direction vector consisting of two arbitrary points inside the structure has a linear combination relation with the same weighting coefficient as the shape transformation formula.

Furthermore, to investigate the change of the line segment $\overrightarrow{a b}$, Equation (14) is obtained by squaring both ends of Equation (13).

$$
|\overrightarrow{a b}|^{2}=w_{0}^{2}\left|\vec{a}_{0} b_{0}\right|^{2}+w_{1}^{2}\left|\vec{a}_{1} b_{1}\right|^{2}+2 w_{0} w_{1}\left|\vec{a}_{0} \vec{b}_{0}\right||| \vec{a}_{1} \vec{b}_{1} \mid \cos \theta
$$

Here, $\theta$ is the angle between the vector $\vec{a}_{0} b_{0}$ and $\vec{a}_{1} \vec{b}_{1}$. Using this formula, it can calculate the length of the line segment after shape transformation. As a special case, when the basis vector becomes parallel, the above equation becomes Equation (15).

$$
|\overrightarrow{a b}|^{2}=w_{0}^{2}\left|\vec{a}_{0} b_{0}\right|^{2}+w_{1}^{2}\left|\vec{a}_{1} b_{1}\right|^{2}+2 w_{0} w_{1}|| \overrightarrow{a_{0} b_{0}}|| \vec{a}_{1} \vec{b}_{1} \mid
$$

To express the length of the line segment, Equation (16) is obtained.

$$
|\overrightarrow{a b}|=w_{0}\left|\vec{a}_{0} b_{0}\right|+w_{1}\left|\vec{a}_{1} b_{1}\right|
$$


Also, when $w_{0}+w_{1}=1$ is substituted, Equation (18) is obtained.

$$
|\overrightarrow{a b}|=\left|\vec{a}_{0} b_{0}\right|
$$

Here, the length of the line segment whose shape has been converted indicates that the original line segment length is always maintained, regardless of the weighting factor.

$$
|\overrightarrow{a b}|=\left|\vec{a}_{0} b_{0}\right|
$$

When using the Equation (18) basis vector method for shape optimization, if the complex shape is decomposed into multiple line segments and holes, and convex-concave are set to the same shape, then in the process of shape optimization-even if the combination of any weight coefficients is converted-the shape will remain unchanged.

\subsection{Proportional Relationship of Distance between Arbitrary Three Points}

Consider the $x$ coordinates of three arbitrary nodes $a, b, c$ inside the structure as an example. Here, between the three nodes $a, b, c$ in the original shape and each basic shape, it is assumed that there is a proportional relationship concerning the same proportional coefficient, respectively.

$$
\frac{x_{a 0}-x_{c 0}}{x_{c 0}-x_{b 0}}=k \frac{x_{a 1}-x_{c 1}}{x_{c 1}-x_{b 1}}=k \frac{x_{a 2}-x_{c 2}}{x_{c 2}-x_{b 2}}=k
$$

These formulas are rewritten to obtain Equation (20),

$$
\begin{gathered}
x_{c 0}=\frac{x_{a 0}+k x_{b 0}}{1+k} \text { (Original shape) } \\
\left.x_{c 1}=\frac{x_{a 1}+k x_{b 1}}{1+k} \text { (Base shape } 1\right) \\
x_{c 2}=\frac{x_{a 2}+k x_{b 2}}{1+k} \text { (Base shape 2) }
\end{gathered}
$$

Also, the $x$ coordinate of the three nodes $a, b$, and $c$ in the newly transformed shape are as follows:

$$
\begin{aligned}
& x_{a}=w_{0} x_{a 0}+w_{1} x_{a 1}+w_{2} x_{a 2} \\
& x_{b}=w_{0} x_{b 0}+w_{1} x_{b 1}+w_{2} x_{b 2} \\
& x_{c}=w_{0} x_{c 0}+w_{1} x_{c 1}+w_{2} x_{c 2}
\end{aligned}
$$

Substituting Equation (20) into the third equation of Equation (21) and rearranging it, Equation (22) is obtained.

$$
x_{c}=\frac{1}{1+k}\left[\left(w_{0} x_{a 0}+w_{1} x_{a 1}+w_{2} x_{a 2}\right)+k\left(w_{0} x_{b 0}+w_{1} x_{b 1}+w_{b 2}\right)\right]
$$

Substituting the first and the second equations of Equation (21) into Equation (22), Equation (23) is obtained.

$$
x_{c}=\frac{x_{a}+k x_{b}}{1+k}, \frac{x_{a}-x_{c}}{x_{c}-x_{b}}=k
$$

This expression is consistent with Equation (20). That is, if the same proportional relationship is set between the original shape and the node coordinates in each basic shape, it shows that the same proportional relationship is always maintained regardless of the weighting coefficient between the nodes in the newly transformed shape.

During the actual shape optimization, if some shapes and their proportional relationship are set in the same way using Equation (23), then even if it is transformed into the combination of arbitrary weight coefficients in the process of shape optimization, the proportional relationship will always remain unchanged. 


\subsection{Holding Relationship of Arbitrary Three Points}

During the case of shape optimization accompanying the rotation of the structure, to investigate the change in the angle of the structure, consider the angle formed by the three nodes $a, b, c$ inside the structure and make the angle between the original shape and the basic shape coincide, as shown in Equation (24).

$$
\theta_{0}=<\overrightarrow{a_{0} b_{0}}, \overrightarrow{a_{0} c_{0}}>=<\overrightarrow{a_{1} \vec{b}_{1}}, \overrightarrow{a_{1} c_{1}}>
$$

Here, $<\overrightarrow{a_{0} \vec{b}_{0}}, \overrightarrow{a_{0} c_{0}}>$ is the angle between the vector $\overrightarrow{a_{0} b_{0}}$ and $\overrightarrow{a_{0} c_{0}}$. Similarly, $<\overrightarrow{a_{1} \vec{b}_{1}}, \overrightarrow{a_{1} c_{1}}>$ is the angle between the vector $\overrightarrow{a_{1} b_{1}}$ and $\overrightarrow{a_{1} c_{1}}$.

Since the aim is to investigate the influence on the angle due to structural rotation, and it is assumed that the side length constituting the angle does not change:

$$
\left|\vec{a}_{0} b_{0}\right|=\left|\vec{a}_{1} b_{1}\right|,\left|\overrightarrow{a_{0} c_{0}}\right|=\left|\overrightarrow{a_{1} c_{1}}\right|
$$

Also, the rotation angle $\Delta \theta$ from the original shape to the basic shape is taken. The vector that constitutes the new angle $\theta$ in the transformed shape is as shown in Equation (26).

$$
\overrightarrow{a b}=w_{0} \vec{a}_{0} \vec{b}_{0}+w_{1} \overrightarrow{a_{1} b_{1}} \overrightarrow{a c}=w_{0} \vec{a}_{0} c_{0}+w_{1} \vec{a}_{1} \vec{c}_{1}
$$

Then, take an inner product on each side of Equation (26):

$$
\overrightarrow{a b} \cdot \overrightarrow{a c}=w_{0}^{2} \vec{a}_{0} b_{0} \cdot \vec{a}_{0} c_{0}+w_{1}^{2} \vec{a}_{1} \vec{b}_{1} \cdot \vec{a}_{1} c_{1}+w_{0} w_{1}\left(\overrightarrow{a_{0} b_{0}} \cdot \overrightarrow{a_{1} c_{1}}+\overrightarrow{a_{1} b_{1}} \cdot \overrightarrow{a_{0} c_{0}}\right)
$$

Considering Equation (25) and rearranging it, Equation (28) can be obtained:

$$
\overrightarrow{a b} \cdot \overrightarrow{a c}=\left|\vec{a}_{0} b_{0}\right|\left|a_{0} c_{0}\right| \cos \theta_{0}\left[1-2 w_{0} w_{1}(1-\cos \Delta \theta)\right]
$$

Conversely, Equation (29) is obtained using the inner product calculation formula of the vector:

$$
\overrightarrow{a b} \cdot \overrightarrow{a c}=|\overrightarrow{a b}||| \overrightarrow{a c} \mid \cos \theta
$$

Making the first equation of Equation (26) square on both sides, Equation (30) can be obtained:

$$
|\overrightarrow{a b}|^{2}=w_{0}^{2}\left|\vec{a}_{0} b_{0}\right|^{2}+w_{1}^{2}\left|\vec{a}_{1} b_{1}\right|^{2}+2 w_{0} w_{1} \vec{a}_{0} \vec{b}_{0} \cdot \overrightarrow{a_{1} b_{1}}
$$

Considering Equation (29) and rearranging it, Equation (31) is obtained:

$$
|\overrightarrow{a b}|^{2}=\left|\vec{a}_{0} b_{0}\right|^{2}\left[1-2 w_{0} w_{1}(1-\cos \Delta \theta)\right]
$$

Likewise, Equation (32) is obtained from the second equation of Equation (26):

$$
|\overrightarrow{a c}|^{2}=\left|\vec{a}_{0} c_{0}\right|^{2}\left[1-2 w_{0} w_{1}(1-\cos \Delta \theta)\right]
$$

By substituting Equations (31) and (32) into Equation (29), Equation (33) is obtained:

$$
\overrightarrow{a b} \cdot \overrightarrow{a c}=\left|\vec{a}_{0} b_{0}\right|\left|a_{0} c_{0}\right| \cos \theta\left[1-2 w_{0} w_{1}(1-\cos \Delta \theta)\right]
$$


By comparing Equations (28) and (33), Equation (34) is obtained:

$$
\cos \theta=\cos \theta_{0}
$$

The new angle $\theta$ in the shape transformed by Equation (34) always coincides with the angle $\theta_{0}$ in the original shape, irrespective of the weighting coefficient.

When actual shape optimization is performed, it is possible to control the change of the angular value composed of three arbitrary points of the structure using Equation (34).

When performing the actual shape optimization using Equation (23), by making the angle value consisting of any three points of the structure in the original shape and in the basis shape identical in a shape optimization way, it can be ensured that the angle value does not ever change.

\section{Shape Optimization Method by Basis Vector}

The shape optimization method shown in Figure 4 can be constructed using the basis vector created by the method of the previous section.

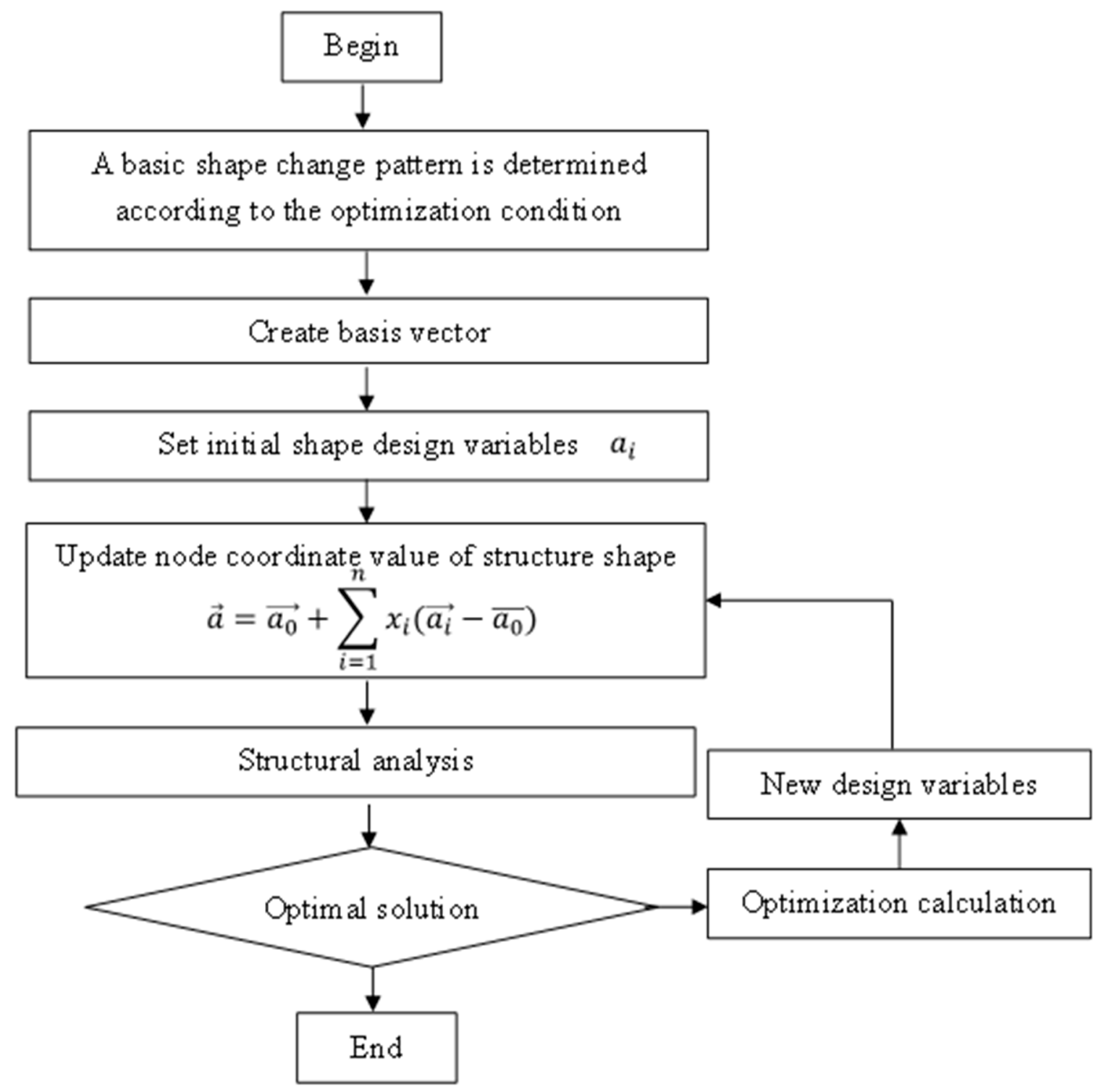

Figure 4. Flow chart of the shape optimization process. 
Shown in Figure 4, at the beginning of the shape optimization after considering the subordinate relationship of partial shapes such as holes and irregularities of the structure, it can determine the basic shape into the basic pattern of shape change. Then, it uses the smoothing method proposed in the previous section for each basic shape to create basis vectors, respectively. Further, from the correspondence relationship between the initial shape and each of the basic shapes, a design variable serving as a weighting factor of the basis vector is obtained. Using Equation (8), the node coordinate value of the structure shape corresponding to the design variable is updated; furthermore, after regular structural analysis and optimization calculations are performed, new design variables are obtained and then substituted into Equation (8) again to update the node coordinate values of the corresponding structure shape, the normal structure analysis and optimization calculation are performed, and the iterative calculation is continued until an optimum solution is obtained.

\section{Application Example}

\subsection{Shape Optimization of Steam Turbine Implantation Section}

The movable wing of the steam turbine for thermal power generation equipment is composed of a blade and a rotor. To replace the blade easily, the root of the tree-type blade is directly inserted into the rotor for assembly, as shown in Figure 5.

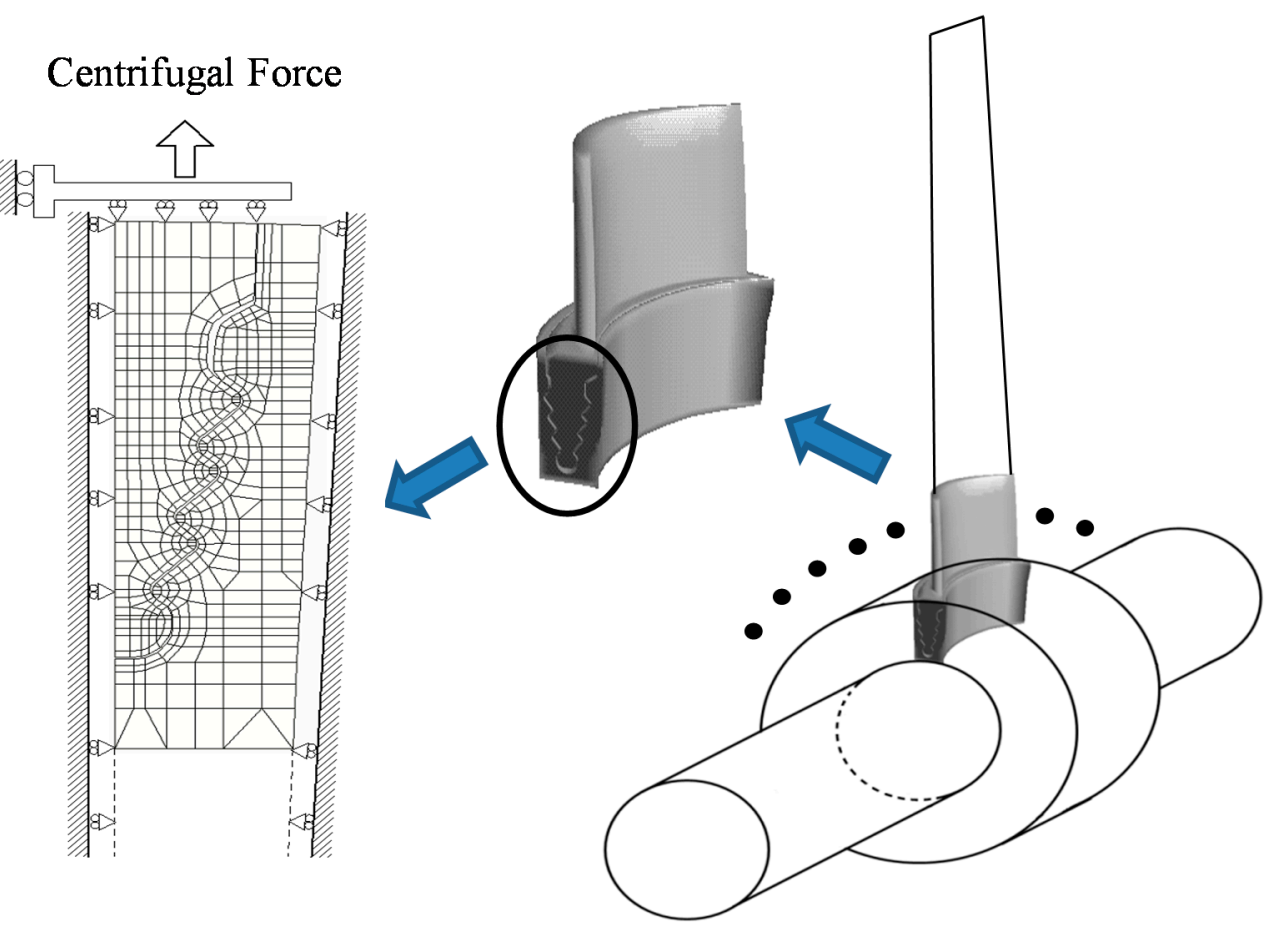

Figure 5. FEM model for fixed part of the blade.

When the steam turbine operates, centrifugal force is created due to the rotation of the rotor action and the concentrated stress is generated in the mutually contacting parts of the tree-type implanted part, which connects the blades and the rotating rotor. When there is improper internal stress in parts, it will seriously reduce the fatigue strength of parts; on the contrary, it will increase the life or fatigue strength of parts.

Since most defects where cracks occur are seen partially, it is necessary to reduce the concentrated stress of the steam turbine as much as possible. How to obtain the optimum shape of the tree-type e implantation part is an important issue.

Here, to simplify the analysis problem, as shown in Figure 5, one blade is cut out from a large number of blades installed along the circumferential direction of the rotating rotor. Further considering 
the periodic symmetry in the circumferential direction, as shown in the figure, half of the blade implanting portion is made into the FEM model, the boundary constraint condition is cyclically symmetrical in the circumferential direction, the centrifugal force is converted from the mass of the blade effective portion, and the rotational speed of the rotor is applied to the root portion of the blade.

The structural design of the implant portion, upon considering the assembled condition of the parts, etc., as the basic pattern of the shape changes, is shown in Figure 6.

$x_{1} \quad$ Changing the slope of the tooth tip

$x_{2} \quad$ Length of implanted part

$x_{3} \quad$ Width of implanted part

$x_{4} \quad$ Width of implanted part

$x_{5}$ Change in the inclination of the tooth contact surface

$x_{6}$ The radius of curvature of the first hook

$x_{7} \quad$ Change in the slope of the first hook
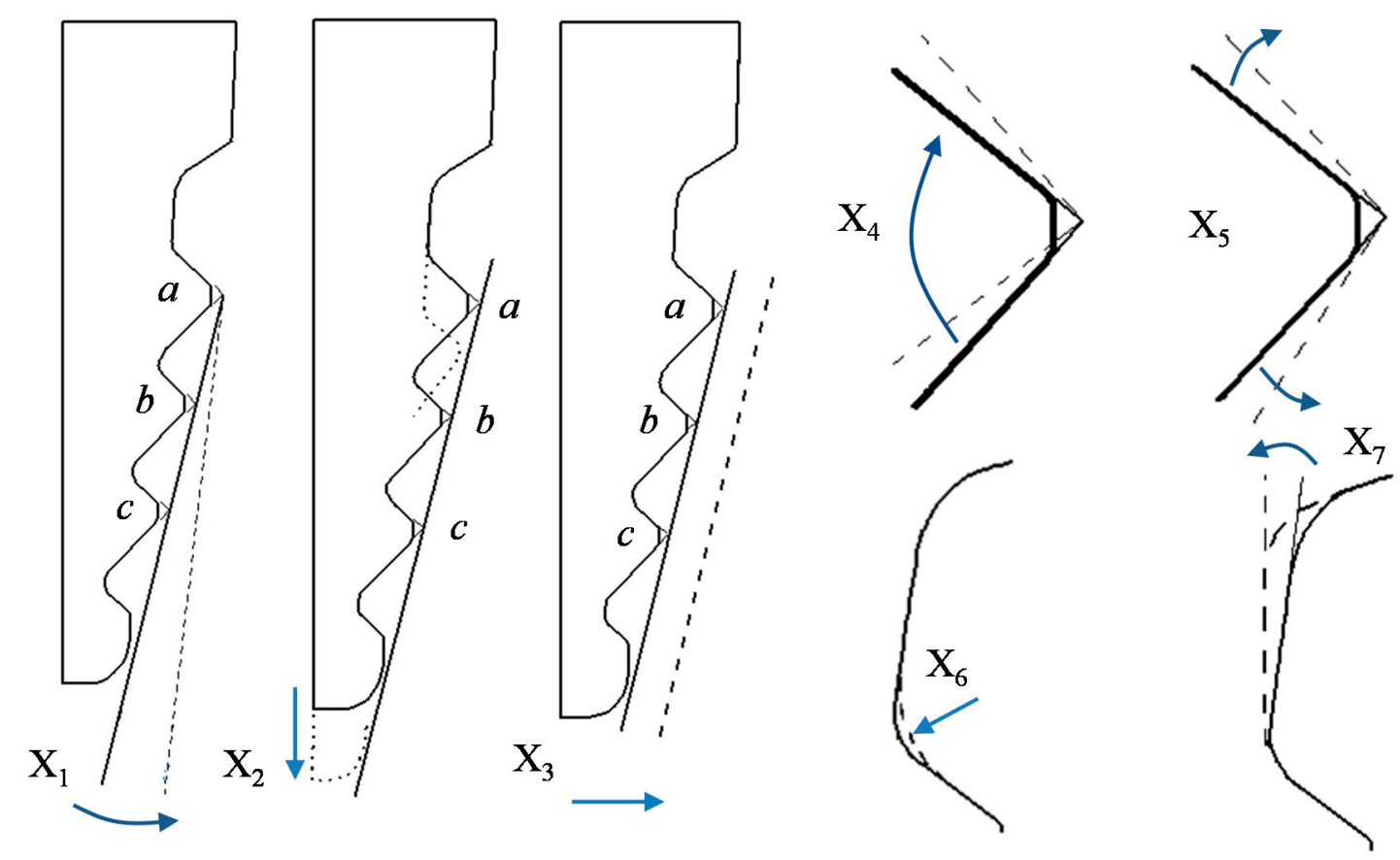

Figure 6. Basis vectors to change shape of fixed part.

Considering the influence of fatigue stress, the constraint condition is the stress difference between the hooks being less than or equal to the allowable value of the stress in each hook, the stress difference between the hooks is added below the allowable value, and the number of constraint conditions is 27 .

However, the seven shape variables shown in Figure 6 cannot be changed freely, there is a partial shape dependency relationship among them, and some shapes should be the same as far as possible. To obtain simple shapes that are easy to process, the shape changes are shown in Figure 6.

(1) Tooth tip points $a, b, c$ are always on the same straight line

(2) The distance between $\overline{a b}$ and $\overline{b c}$ of a tooth tip point are always equal

(3) The curved contour line of each hook is always the same

An optimum shape will be determined on the premise that the requirements relating to these three shape changes are forcibly satisfied.

To realize this problem, following the shape indicated by the basic shape change pattern shown in Figure 7 and using the smoothing method discussed in the previous section to generate a basis vector, shape optimization can be performed according to the algorithm shown in Figure 4. 


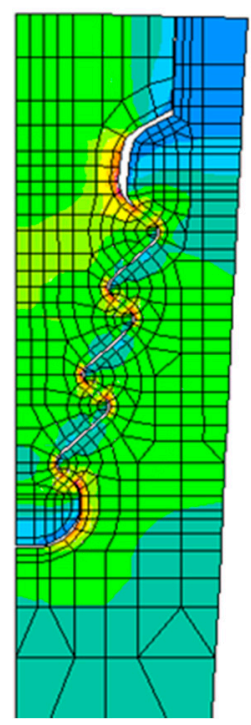

(a). Initial shape

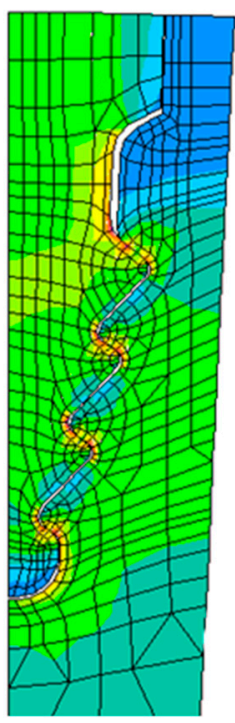

(b). Optimum Shape

Figure 7. Shape and stress distribution of fixed part of blade.

Aiming for the optimization constraint condition, the maximum mises stress value on the blade side and the rotor side in each hook is limited to the allowable amount or less. Furthermore, constraints are added to the stress difference between the hooks so stress is not concentrated on one hook, but dispersed over the whole. The number of constraint conditions is 27 in total.

Figure 7 shows the stress distribution of the optimum shape and initial shape obtained by optimization calculation. Considering Figure 7, a relatively low distribution is seen in the upper part on the rotor side and the lower part on the blade side in the stress distribution of the initial shape. As a result of averaging the load sharing rate by optimization, the tooth tip inclination angle becomes larger with respect to the vertical plane, and the lateral width of the moving blade increases.

Since the shape and centrifugal force of the blade implant are limited, the average stress value is determined by the ratio of centrifugal force and cross-section, so it is difficult to reduce the comprehensive stress level only by adjusting the internal shape. Therefore, the purpose of the optimization problem is to balance the stress distribution in the implant.

Accompanying the increase of the width and the movement of the material, the stress on the blade side is lower. Since the stress on the rotor side tends to be higher, the angle of the contact surface becomes acute relative to the horizontal plane, and the angle of the tooth surface clamp becomes larger. Additionally, to reduce the stress concentration of the first hook, the stress balance should be maintained to increase the radius of curvature.

Table 1 shows the change in the basis vector coefficient corresponding to each shape change pattern. The change range of each coefficient is from -1 to 1 , and the initial value is 0 . Regarding the optimum shape, it coincides with the shape shown in Figure 6 where the coefficient becomes 1 , is adopted $100 \%$, and the coefficient becomes minus changes in the direction opposite to the shape shown in Figure 6. 
Table 1. Change of Design Variable Coefficients.

\begin{tabular}{ccccc}
\hline Variable & Low Limit & Up Limit & Initial & Optimal \\
\hline $\mathrm{X}_{1}$ & -1.00 & 1.00 & 0.00 & -0.401 \\
\hline $\mathrm{X}_{2}$ & -1.00 & 1.00 & 0.00 & 1.000 \\
\hline $\mathrm{X}_{3}$ & -1.00 & 1.00 & 0.00 & 0.745 \\
\hline $\mathrm{X}_{4}$ & -1.00 & 1.00 & 0.00 & -0.573 \\
\hline $\mathrm{X}_{5}$ & -1.00 & 1.00 & 0.00 & 0.214 \\
\hline $\mathrm{X}_{6}$ & -1.00 & 1.00 & 0.00 & 1.000 \\
\hline $\mathrm{X}_{7}$ & -1.00 & 1.00 & 0.00 & 0.956 \\
\hline
\end{tabular}

Figure $8 \mathrm{a}$ is a distribution showing the Mises stress value for the initial shape and the optimum shape along with the surface layer of the blade. The vertical axis is the stress value, and the hook corresponding to the horizontal axis is shown in Figure 3. Similarly, Figure $8 \mathbf{b}$ shows the stress distribution along with the surface layer on the rotor side. Going from there, the concentrated stress of the first hook on the blade side and the fourth hook on the rotor side is reduced, in which stress is high in the initial shape. Additionally, it is found that the maximum stress at each hook is almost the same value, the stress difference of each hook is decreased, and the overall stress balance is improved.

Further, as shown in Figure 7, it is understood that the assembling dimension of the blade implant portion (the distance between the hook and the hook) is not changed as compared with the original. After optimization, it is easy to confirm that the size of the position of the hook coincides with the original. That is, the position of the vertex of each hook becomes a straight line, and all three requirements for shape change are satisfied.

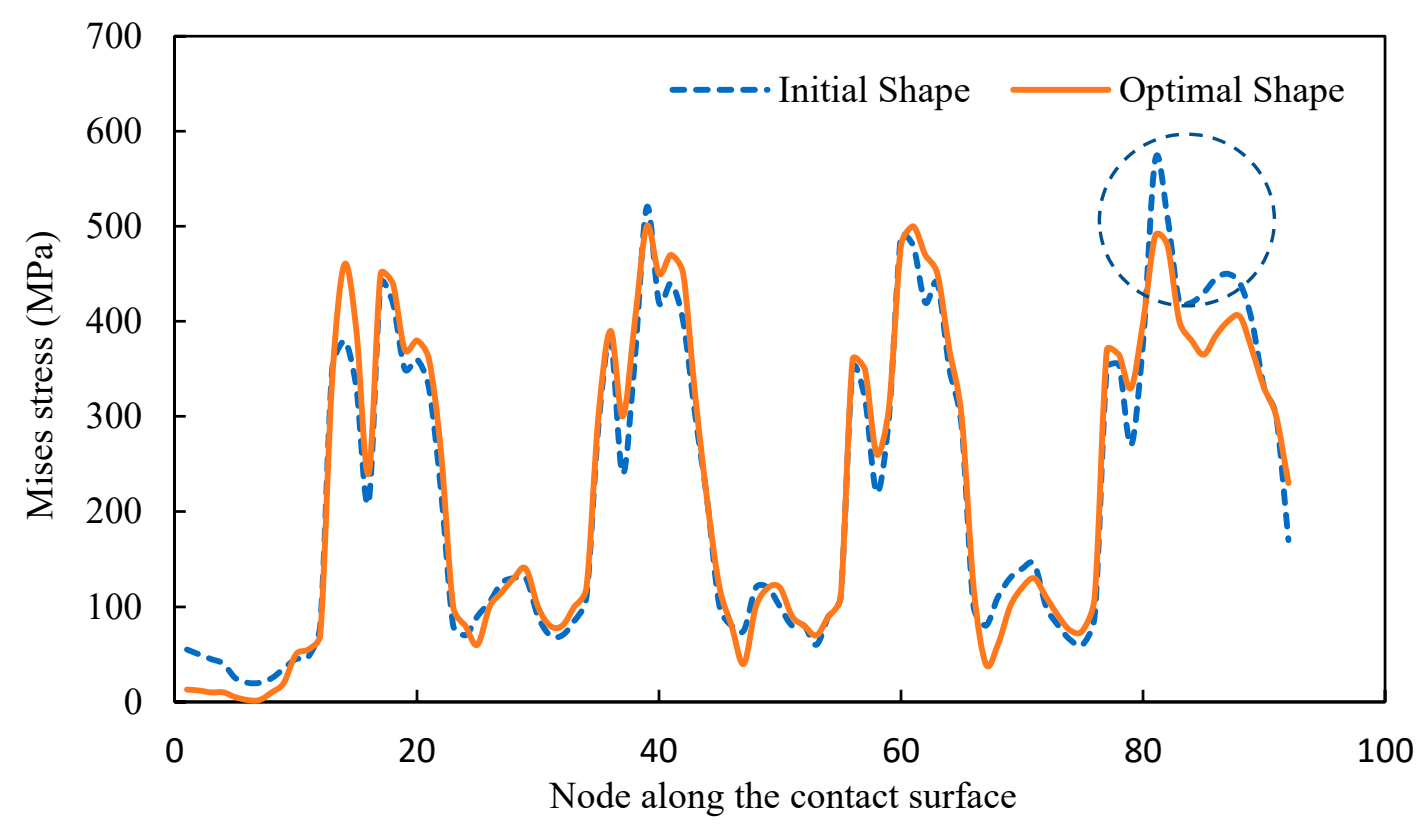

(a)

Figure 8. Cont. 


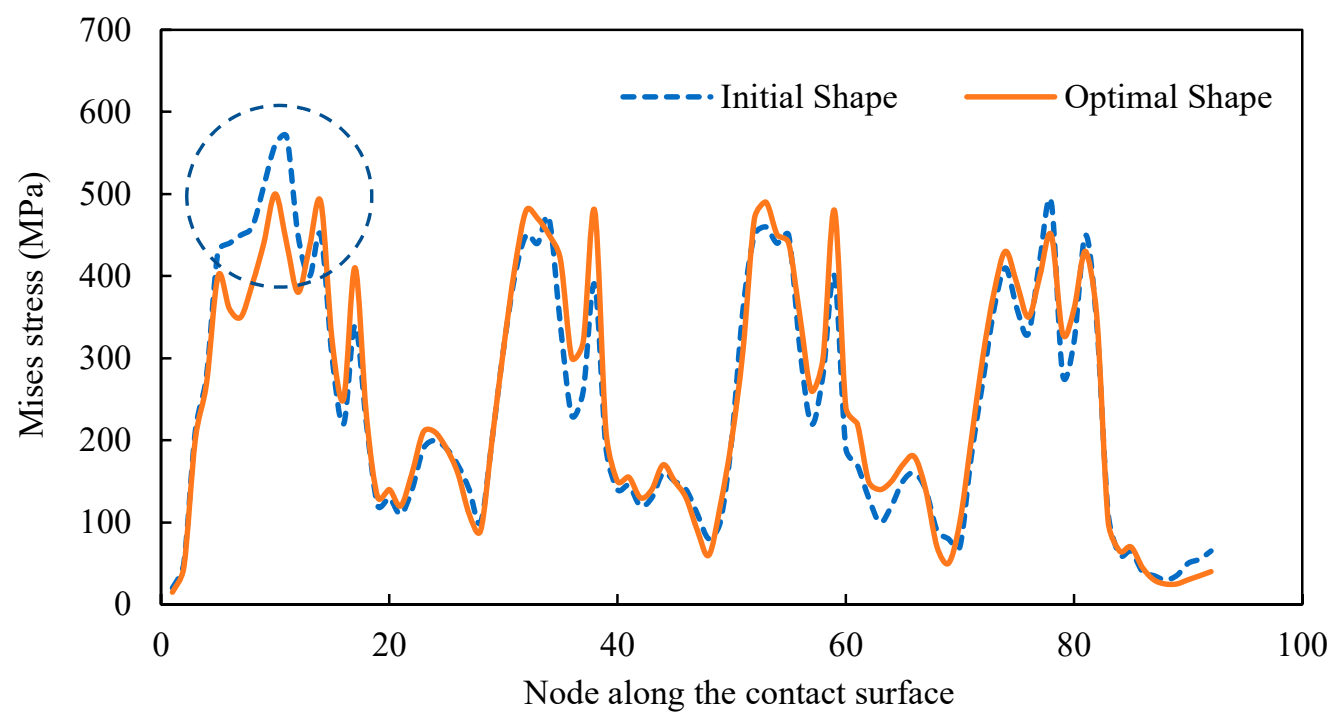

(b)

Figure 8. Stress distribution along face of blade side and rotor side. (a) Stress distribution along face of blade side, (b) Stress distribution along face of rotor side.

\subsection{Stress Distribution Optimization of the Engine Mount Bracket}

Figure 9 shows a model of an engine mount bracket for fixing a passenger car engine. The load conditions occur when applying sudden braking, and the uniform load in the oblique direction is applied along the lower half inner diameter of the upper hole. The value of the uniform load is $6000 \mathrm{~N}$ in the axial direction, $23,000 \mathrm{~N}$ in the lateral direction, and 45,000 $\mathrm{N}$ in the downward direction.

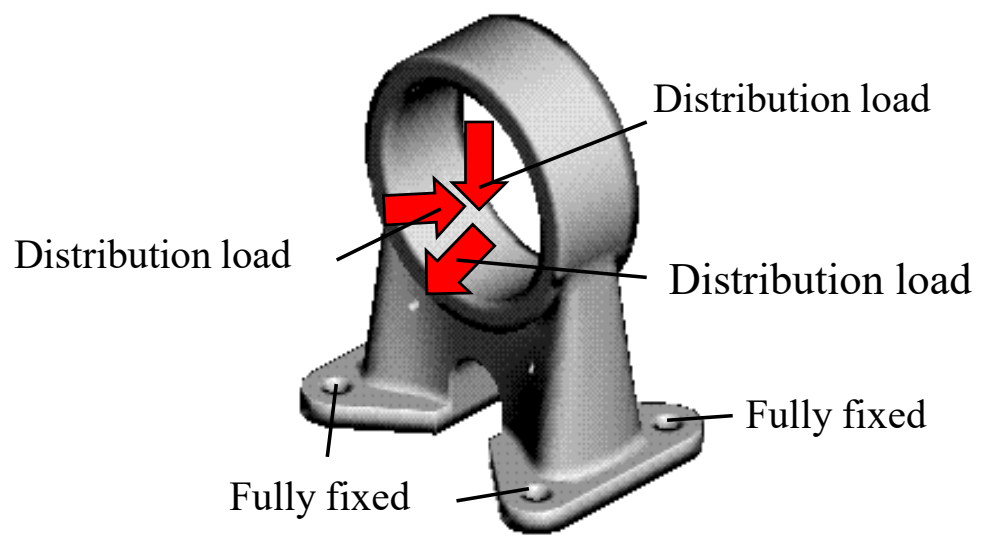

Figure 9. An engine mount bracket for fixing a passenger car engine.

The dotted line in Figure 10 represents the range in which the structural shape under design can be changed and, in the optimization calculation, these change ranges represent the basis vectors. Furthermore, by adjusting the ratio and combination of each basis vector, the optimum shape can be obtained.

The objective function of the optimization calculation minimizes the weight of the structure. Concerning the constraint condition, the maximum stress of each part is set to the allowable stress value of $140 \mathrm{MPa}$ or less. Optimum solutions are obtained by optimization, and the optimal parameters corresponding to the shape change pattern represented by the dotted line in Figure 10 are shown in Table 2. Regarding the table, column 1 is the shape parameter, column 2 is the maximum value of the shape parameter, column 3 is the initial value of the shape parameter, column 4 is the optimum value of the shape parameter, and column 5 is the adoption rate of the shape change pattern. 
Table 2. Change of design variables $(\mathrm{mm})$.

\begin{tabular}{ccccc}
\hline & Maximum & Initial & Optimum & Adopt. (\%) \\
\hline $\mathrm{X}_{1}$ & 5.0 & 10.0 & 6.9 & $62 \%$ \\
\hline $\mathrm{X}_{2}$ & 8.4 & 0.0 & 8.4 & $100 \%$ \\
\hline $\mathrm{X}_{3}$ & 18.0 & 0.0 & 18.0 & $100 \%$ \\
\hline $\mathrm{X}_{4}$ & 54.0 & 45.0 & 52.9 & $88 \%$ \\
\hline $\mathrm{X}_{5}$ & 25.0 & 15.0 & 20.0 & $50 \%$ \\
\hline $\mathrm{X}_{6}$ & 60.0 & 30.0 & 57.0 & $90 \%$ \\
\hline $\mathrm{X}_{7}$ & 6.6 & 0.0 & 4.6 & $70 \%$ \\
\hline $\mathrm{X}_{8}$ & 7.0 & 0.0 & 7.0 & $100 \%$ \\
\hline $\mathrm{X}_{9}$ & 5.4 & 0.0 & 5.4 & $100 \%$ \\
\hline
\end{tabular}
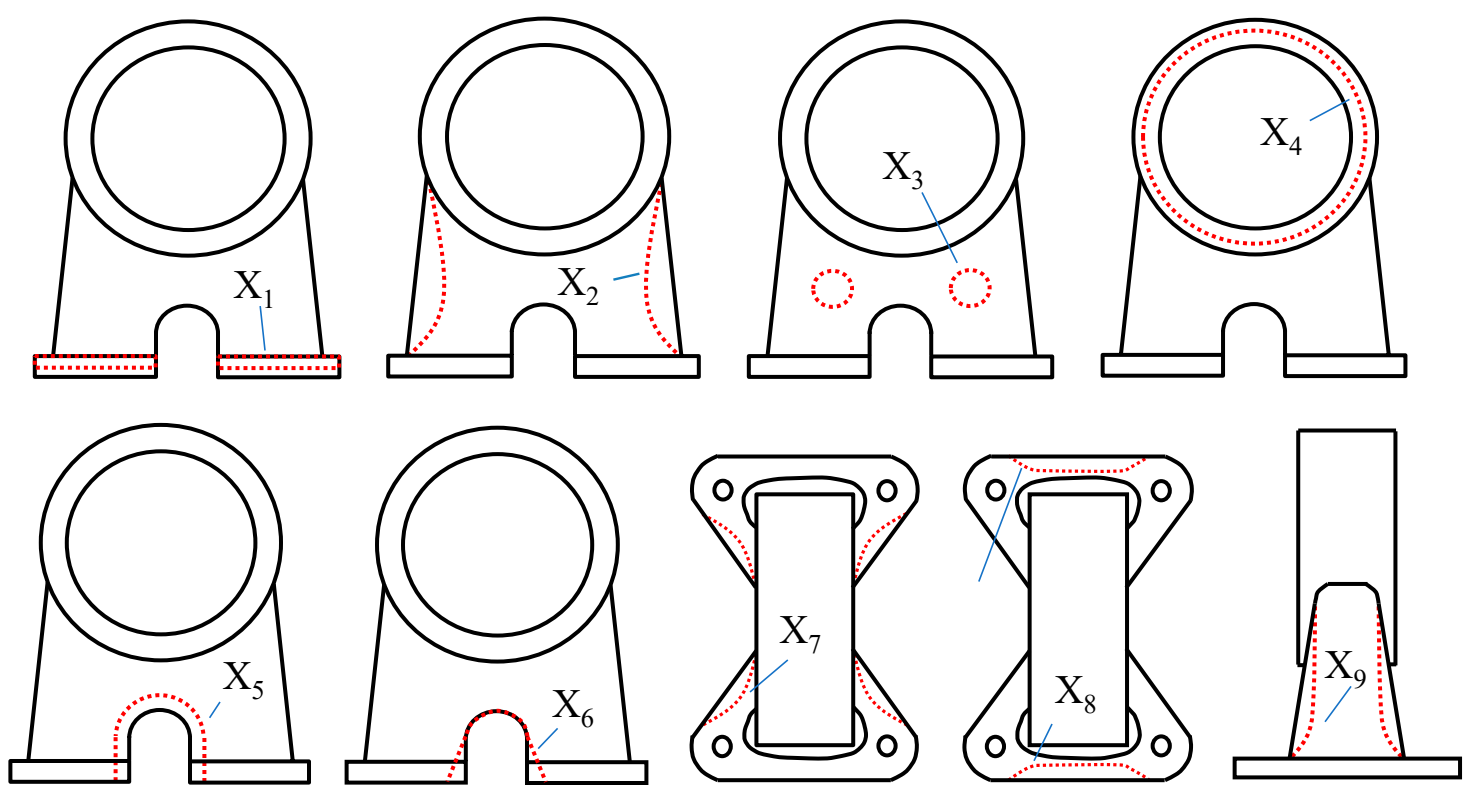

Figure 10. The structure and shape change area.

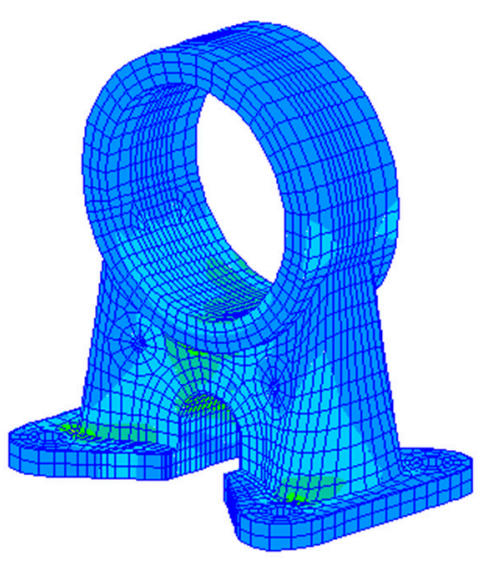

(a)

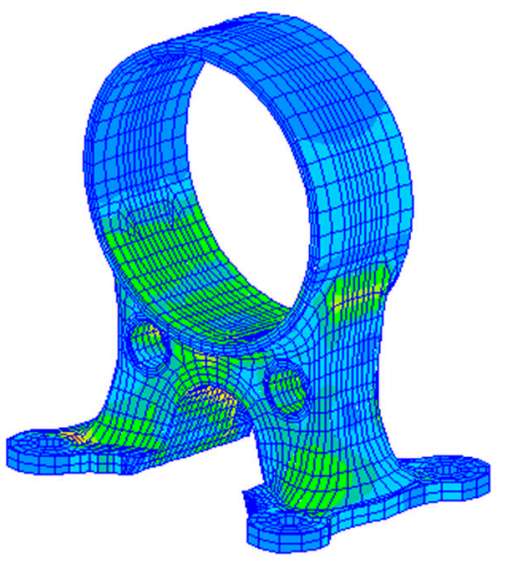

(b)

Figure 11. Stress distributions of engine mount bracket. (a) Initial shape, (b) Optimum Shape. 
Considering the stress distribution diagram shown in Figure 11, in the optimization calculation, each of these change ranges is represented by a basis vector. Concerning the original shape, the maximum Mises stress is $98.4 \mathrm{MPa}$, and there is a significant difference from the allowable value, which shows that useless material is used more than necessary. Regarding the optimized shape, the maximum Mises stress is $136.8 \mathrm{MPa}$ higher than that of the original shape. Still, the difference from the allowable value is small, the stress in each part is dispersed, and the performance of the material can be fully utilized.

Compared to the original in Figure 11, concentrated stress is reduced and stress is dispersed. Weight decrease, as the objective function, is $49.9 \%$ lighter than the original shape. Additionally, it shows that the requirements for the relative relationship between the external boundary shape and the inner hole are all satisfied and the partial shape subordination problem is solved.

\section{Discussion}

Based on the results of the examination of the practical example in the previous section, it is shown that it is effective to solve the shape optimization problem having the partial shape subordinate relation using the method proposed in this report. Regarding the case applying to the problem, it also is necessary to examine several problems in detail.

\subsection{Creation of Basis Vectors}

Prior to shape optimization using the basis vector method, it is necessary to create a basic shape change pattern as a reference for the shape change and a basis vector represented by its node coordinates. Additionally, compared to the original shape, the basic shape is required to be the same as that of the original model, and only the coordinate values of the basis vector and the original vector having the same node number are different from each other.

Here, the paper proposed a basis vector creation method making full use of the finite element method analysis mesh smoothing technique. Specifically, as explained using the simple example shown in Figure 12, a basis vector can be created according to the following procedure:

(1) According to the design scheme of the original shape, the analytical model used to generate the basic vector is divided into three parts: the fixed part $\left(a_{1}, a_{2, \prime}, a_{5}\right)$, the specified part $\left(b_{1}, b_{2, \prime}, b_{5}\right)$, and other shape-related changes as shown in Figure 12a.

(2) A new node coordinate is given to the designated part $\left(b_{1}, b_{2, \prime}, b_{5}\right)$ of the shape change, and the node position of the designated part is updated as shown in Figure $12 \mathrm{~b}$.

(3) To enable the shape-related change part, excluding the fixed part $\left(a_{1}, a_{2}, \ldots, a_{5}\right)$ whose shape is not changed and the designation part $\left(b_{1}, b_{2, \prime}, b_{5}\right)$ to forcibly change, a smoothing calculation of the analysis mesh is performed using Equation (35).

$$
x_{i}=\frac{1}{N} \sum_{j=1}^{J} x_{j} y_{i}=\frac{1}{N} \sum_{j=1}^{J} y_{j} z_{i}=\frac{1}{N} \sum_{j=1}^{J} z_{j}
$$

Considering Equation (35), $x_{i}, y_{i}, z_{i}$ are the new coordinate values of the nodes $i$ of the shape-related change part, $x_{i}, y_{i}, z_{i}$ are the neighboring node $i$ coordinate values adjacent to the node, and $j$ is a surrounding node-set adjacent to nodes $i$.

(4) Smoothing calculation of the mesh, with respect to the repetitive shape-related change part, is performed using Expression (35) and, as a result, a smooth basis shape can be obtained. Here, the basis shape obtained by the number of repetitive smoothing calculations is also different. The basic shape can be finely adjusted according to the actual shape optimization request so the number of repetitive calculations is small in Figure 12c, and the shape with many repetitive calculations is shown in Figure 12d.

(5) Basis vectors can be created directly by sequentially arranging the node coordinate values of the basic shape obtained last. 
When actually generating a basis vector there are two methods; one method is moving a shape designation portion directly, as shown in Figure 13, and the other is a projection method to a CAD shape in which a shape designation portion is accurately created, as shown in Figure 14.

Figure 13a shows an original shape in which a circular hole is drilled in the center of a thick square plate, and Figure $13 \mathrm{~b}$ is a basic shape change pattern that moves only the shape designation portion around the inner hole in the upward direction.

Figure 14a shows an original shape having a thick-walled cylindrical shape, the inner surface of which is a fixed portion, and the outer surface is a shape designating portion. Shown in Figure 14b, a CAD shape to be the destination of the shape designation portion is prepared.

Figure $14 \mathrm{c}$ shows the outer surface of the cylinder is projected to the CAD shape, at the same time, the analysis mesh of the thick portion of the cylinder is subjected to smoothing processing and, finally, a basic shape change pattern is shown in Figure 14d.

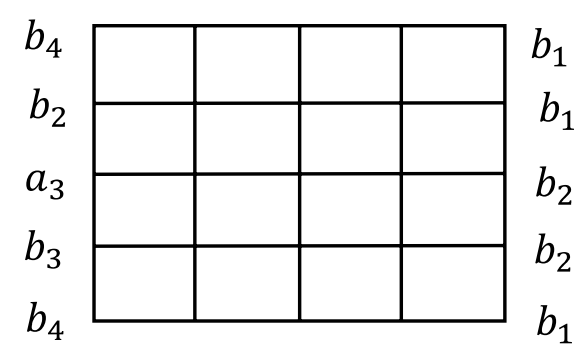

(a)

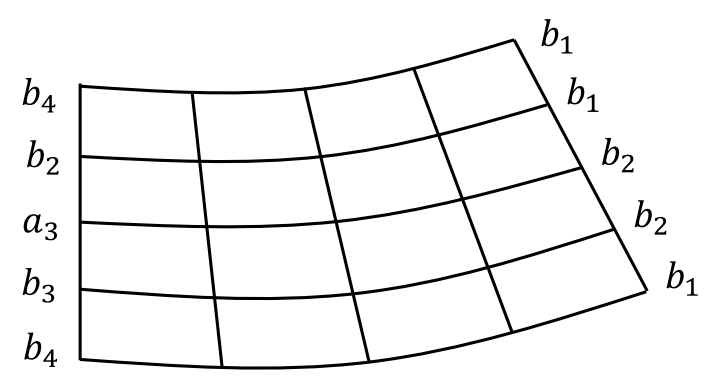

(c)

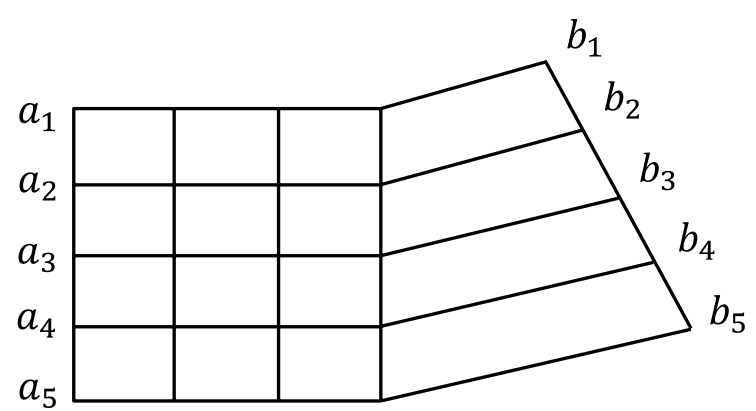

(b)

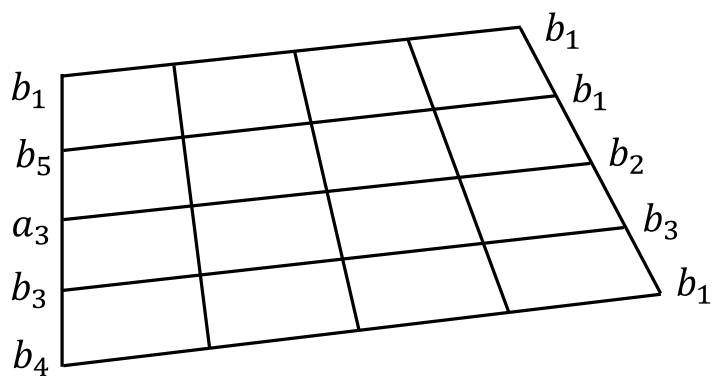

(d)

Figure 12. Creation of basis vectors.

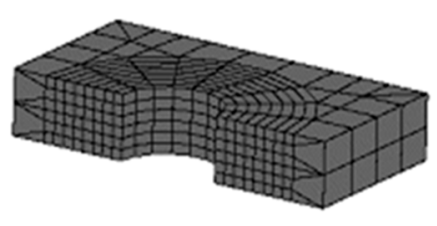

(a)

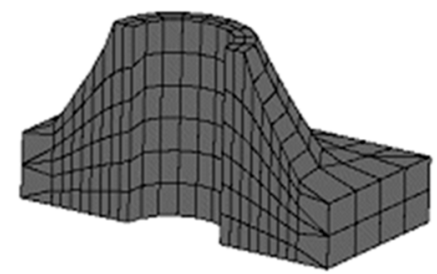

(b)

Figure 13. A shape designation portion. 


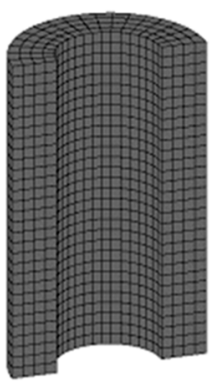

(a)

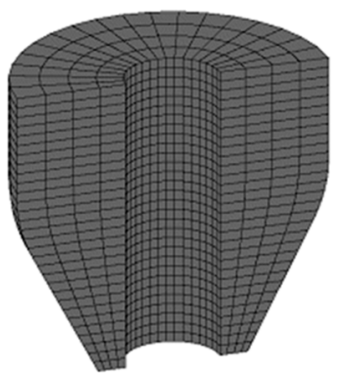

(c)

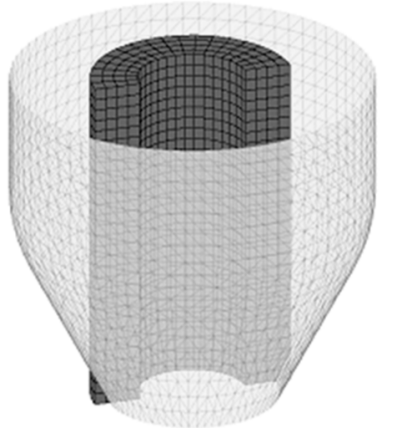

(b)

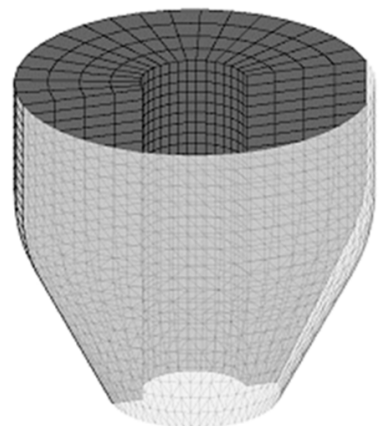

(d)

Figure 14. An original shape having a thick walled cylindrical shape.

\subsection{Mutual Independence Problem of Basis Vectors}

When changing the structure shape using the shape transformation Equation (3), for the combination of arbitrary weighting coefficients $w_{i}$, the shape vector obtained by calculation using Equation (7) is considered to be represented by a linear combination of the basis vectors. Here, the mutual relationship between each basis vector is considered.

Figure 15 shows a structure composed of two elements, and its nodes 1, 2, and 3 are defined as shape change nodes. The basis vectors corresponding to the original shape and the basic shape change pattern shown in Figure 15a-d are expressed by Equations (36)-(39).

$$
\begin{aligned}
& \alpha_{0}=\{10,40,10,20,10,0\}^{T} \\
& \alpha_{1}=\{30,40,20,20,10,0\}^{T} \\
& \alpha_{2}=\{10,40,20,20,30,0\}^{T} \\
& \alpha_{3}=\{20,40,20,20,20,0\}^{T}
\end{aligned}
$$

Further, the shape change vector represented by the difference between the basic shape change pattern and the basis vector of the original shape is expressed by Equation (37).

$$
\begin{aligned}
& \beta_{1}=\alpha_{1}-\alpha_{0}=\{20,0,10,0,0,0\}^{T} \\
& \beta_{2}=\alpha_{2}-\alpha_{0}=\{0,0,10,0,20,0\}^{T} \\
& \beta_{3}=\alpha_{3}-\alpha_{0}=\{10,0,10,0,10,0\}^{T}
\end{aligned}
$$

Here, to evaluate the correlation between the two shape change vectors $\beta_{1}$ and $\beta_{2}$, it is necessary to evaluate using the MAC (Modal Assurance Criterion) value calculated using the $M A C\left(\beta_{1}, \beta_{2}\right)$ value calculation, Equation (38), used for evaluating the mutual relationship of the vibration modes.

$$
\operatorname{MAC}\left(\beta_{1}, \beta_{2}\right)=\frac{\left(\beta_{1}^{T} \beta_{2}\right)^{2}}{\left(\beta_{1}^{T} \beta_{1}\right)\left(\beta_{2}^{T} \beta_{2}\right)}
$$




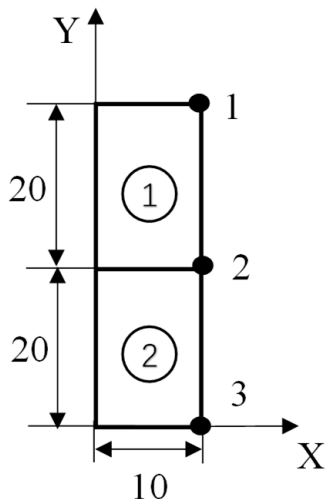

(a)

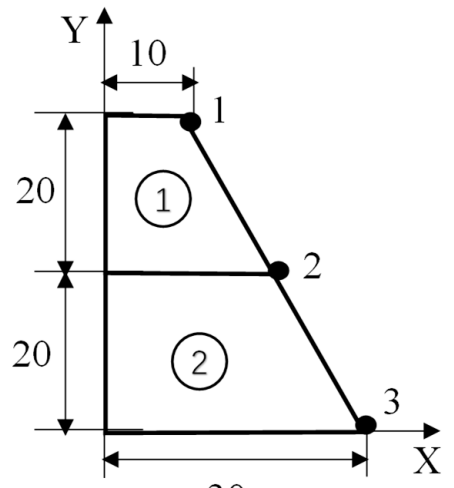

30

(c)

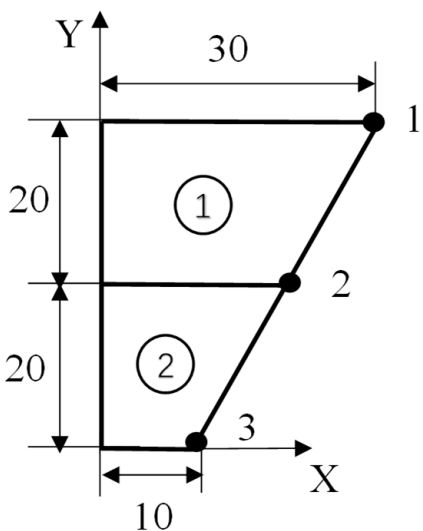

(b)

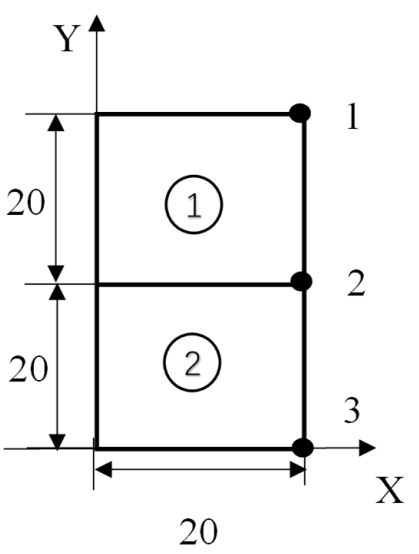

(d)

Figure 15. An example for independence of basis vectors. (a) Original shape, (b) Basis shape 1, (c) Basis shape 2, (d) Basis shape 3.

Using Equation (38), it is understood that $0 \leq M A C\left(\beta_{1}, \beta_{2}\right) \leq 1$. When $M A C\left(\beta_{1}, \beta_{2}\right)=1$ shape change vectors $\beta_{1}$ and $\beta_{2}$ have a linearly independent relationship, it indicates that there is no mutual influence between shape change patterns represented by $\beta_{1}$ and $\beta_{2}$. When $\operatorname{MAC}\left(\beta_{1}, \beta_{2}\right)=0$ it indicates that there is a proportional relation $\beta_{1}=K \beta_{2}$ between shape change vectors $\beta_{1}$ and $\beta_{2}$, and eliminating one in $\beta_{1}$ and $\beta_{2}$ does not affect the shape optimization result.

Therefore, with respect to the shape change vector of Equation (37), the MAC value between each shape change vector is calculated using Equation (38), and the result becomes Equation (39).

$$
\operatorname{MAC}\left(\beta_{1}, \beta_{2}\right)=0.04 \operatorname{MAC}\left(\beta_{2}, \beta_{3}\right)=0.6 \operatorname{MAC}\left(\beta_{1}, \beta_{3}\right)=0.6
$$

Considering the case of shape optimization by the basis vector method, it is ideal if there is a mutual linear independent relationship between each shape change vector; however, as shown in Figure 7, when a plurality of basic shape change patterns are set along the same direction with respect to one boundary shape, as shown in Equation (39), a certain degree of change- even though there is a linear relationship since it is possible to express shape change accurately by a combination of shape change vectors-is verified by the results of numerical analysis that the result of shape optimization is hardly affected.

However, if the linear combination between the shape change vectors is potent and the MAC value is too close to 1 , then the design sensitivity components corresponding to the design variable may interact with each other. When the corresponding basic shape change modes are adjusted, for example, 
two basic shape change modes are changed to one. It can greatly improve the analytical efficiency and quality of shape optimization.

\subsection{Remeshing Problem of Analysis Model}

During the process of shape optimization, with the change of shape, the mesh of the analysis model will change greatly. The aspect ratio of some meshes, or the inner angle of the element edge, will be increased so the mesh quality will have significantly deteriorated, so it may not be able to continue the analysis in the process of shape optimization.

To solve this problem, when each basic shape change pattern is determined and the corresponding basis vector is created, the mesh quality corresponding to each basis vector can be checked using the following Equation (40):

$$
\alpha=\alpha_{0}+w_{i}\left(\alpha_{i}-\alpha_{0}\right) i=1,2,, n
$$

Here $\alpha_{0}$ and $\alpha_{i}$ are the initial shape and the basis vector corresponding to the basic shape change pattern, respectively, $w_{i}$ is the weight coefficient corresponding to the $i$ th basic shape change pattern, and $n$ is the number of basis vectors.

A concrete method of checking the analysis mesh will be described using the simple example shown in Figure 16a-c which are structural shapes and analysis meshes obtained by setting weighting coefficients $w_{i}$ to $w_{i}=0$, a maximum value $\left(w_{i}=1\right)$, and a minimum value $\left(w_{i}=-1\right)$, respectively. Also, the weighting coefficient $w_{i}$ may be considered as the adoption rate of the $i$ th basic shape change pattern, but $w_{i}=1$ and $w_{i}=-1$ mean that the respective basic shape change pattern is adopted.

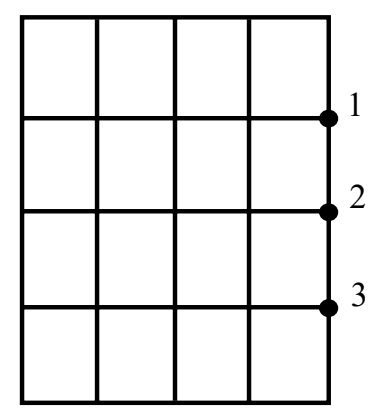

(a)

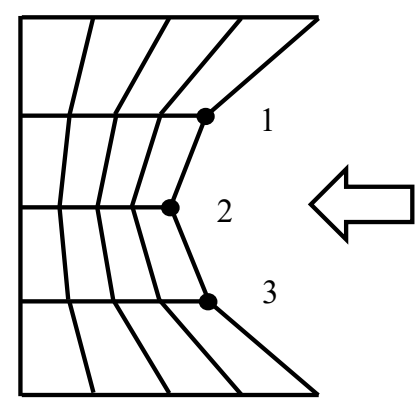

(b)

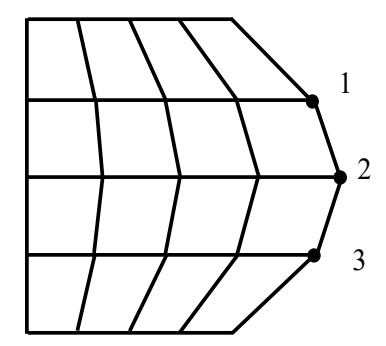

(c)

Figure 16. Remeshing problem of analysis model, (a) $w_{i}=0,(\mathbf{b}) w_{i}=1,(\mathbf{c}) w_{i}=-1$.

Here, only the analysis mesh of Figure 16b,c with the largest shape change is checked with respect to the analysis mesh of the original shape and, if there is no problem in the quality of the analysis mesh of these two cases, all of the analysis meshes of the structural shapes formed in the optimization process linearly change between Figure $16 \mathrm{~b}, \mathrm{c}$, so there is no problem in the quality of the analysis mesh.

When there is a problem with the quality of the analytical grid in Figure 16b,c, if the analysis fails, the maximum or minimum value of the weighting coefficient can be adjusted appropriately in the direction close to 0 , or the basic shape change mode $i$ is modified, and the system vector can be recreated according to the situation.

\section{Summary and Conclusions}

While analyzing the three-dimensional shape optimization problem, the following conclusions were obtained in detail during the study of the internal shape control problem, the so-called partial shape subordination problem with shape change of the external structure boundary.

1. The partial shape subordination problem was classified into three basic modes: the corresponding relation of the line segment formed by any two points, the proportional relationship of the distance 
between any three points, and the maintaining relationship of the angle formed by any three points. It was theoretically proved that the method of controlling the internal shape correctly with the change of the shape of the external boundary was used in the analysis of complex structural shape optimization problems. Thus, the problem of partial shape subordination which has not been adequately solved.

2. To solve the shape optimization problems of complex structures, including some shape related problems, the basic vector method was proposed, and an actual optimization example was used to verify the proposed optimization method. The results show that the optimization method was effective for the partial shape subordination problem in complex structural shape optimization problems.

3. Regarding the most important reference vector-making problem in the application of a reference vector, the study put forward the method of making a reference vector based on the smoothing method, and made a reference vector correctly according to the requirements of optimization design. Additionally, the combination of CAD shape corresponding to the basic shape change mode was applied to more practical optimization problems.

4. To use the linear combination of multiple reference vectors to represent the shape change of shape optimization, before the implementation of shape optimization, the relationship between the reference vectors was studied. The MAC value used in evaluating the relationship between vibration modes was used, and the reference vectors were linear. The method of quantity was confirmed to be helpful in improving the stability of the analysis and calculation of complex shape optimization problems.

5. During the process of shape optimization analysis, along with the change of the structure shape, the resolution reset changed greatly, and the quality of some analytic grids was significantly reduced. To avoid the problem where the normal structural analysis could not be continued, before the implementation of shape optimization and to avoid that every basic shape change pattern will have an analytic grid, the study proposed a checking method and remeshing method to solve the problem. During the process of shape optimization calculation, the degradation of the analysis grid resulted in the decrease of calculation.

6. Here, two typical cases were verified by the basic vector method. Similar problems with the case are very common in practice. The research results of this paper can be well verified from the two typical cases (steam turbine and engine mount bracket). It was seen from the result that the stress value of the final optimization result (solid line) was low, and the stress value of each point was uniformly distributed, which avoided the occurrence of stress concentration. Taken from this result, the effectiveness of the basic vector method proposed in this paper was well proved. This method can be well solved, which is of great help to actual engineering problems.

Based on the results of this research, the partial shape dependency problem was able to be solved and it made a step forward toward a practical application that effectively solves the complicated structure optimization problem frequently found in the design site.

Author Contributions: Q.W. contributed to study concepts, study design and manuscript preparation, H.Z. and W.Z. contributed to data acquisition and manuscript editing, X.Z. contributed to guarantor of integrity of entire study. All authors have read and agreed to the published version of the manuscript.

Funding: This research received no external funding.

Conflicts of Interest: The authors declare no conflict of interest.

\section{References}

1. Ma, Z.-D.; Kikuchi, N.; Hagiwara, I. Structural topology and shape optimization for a frequency response problem. Comput. Mech. 1993, 13, 157-174. [CrossRef]

2. Wang, W.; Rivard, H.; Zmeureanu, R. Floor shape optimization for green building design. Adv. Eng. Inform. 2006, 20, 363-378. [CrossRef] 
3. Azegami, H.; Takeuchi, K. A smoothing method for shape optimization: Traction method using the robin condition. Int. J. Comput. Methods 2006, 3, 21-33. [CrossRef]

4. Yoon, M.; Lee, J.; Koo, B. Shape design optimization of thermoelasticity problems using isogeometric boundary element method. Adv. Eng. Softw. 2020, 149, 102871. [CrossRef]

5. He, D.; Cao, Y.; He, D.; Sun, S. Optimized design of composite grating in real-time three-dimensional shape measurement. Optik 2015, 126, 2781-2787. [CrossRef]

6. Leary, M.; Merli, L.; Torti, F.; Mazur, M.; Brandt, M. Optimal topology for additive manufacture: A method for enabling additive manufacture of support-free optimal structures. Mater. Des. 2014, 63, 678-690. [CrossRef]

7. Vatanabe, S.L.; Lippi, T.N.; De Lima, C.R.; Paulino, G.H.; Silva, E.C. Topology optimization with manufacturing constraints: A unified projection-based approach. Adv. Eng. Softw. 2016, 100, 97-112. [CrossRef]

8. Lu, J.; Chen, Y. Manufacturable mechanical part design with constrained topology optimization. Proc. Inst. Mech. Eng. Part B J. Eng. Manuf. 2012, 226, 1727-1735. [CrossRef]

9. Chiandussi, G.; Codegone, M.; Ferrero, S.; Varesio, F.E. Comparison of multi-objective optimization methodologies for engineering applications. Comput. Math. Appl. 2012, 63, 912-942. [CrossRef]

10. Kirthana, S.; Nizamuddin, M.K. Finite Element Analysis and Topology Optimization of Engine Mounting Bracket. Mater. Today Proc. 2018, 5, 19277-19283. [CrossRef]

11. Kolhapure, R.; Rawal, M.; Rayjadhav, S.; Kabnure, B.; Mekalke, G.; Patil, R.; Balwan, V. Shape optimization of washer load cell using GRA method. Mater. Today Proc. 2020, 27, 238-243. [CrossRef]

12. Mam, K.; Douthe, C.; Le Roy, R.; Consigny, F. Shape optimization of braced frames for tall timber buildings: Influence of semi-rigid connections on design and optimization process. Eng. Struct. 2020,216, 110692. [CrossRef]

13. Mantovani, S.; Presti, I.L.; Cavazzoni, L.; Baldini, A. Influence of Manufacturing Constraints on the Topology Optimization of an Automotive Dashboard. Procedia Manuf. 2017, 11, 1700-1708. [CrossRef]

14. Mayencourt, P.; Mueller, C. Hybrid analytical and computational optimization methodology for structural shaping: Material-efficient mass timber beams. Eng. Struct. 2020, 215, 110532. [CrossRef]

15. Tang, Y.; Mak, K.; Zhao, Y.F. A framework to reduce product environmental impact through design optimization for additive manufacturing. J. Clean. Prod. 2016, 137, 1560-1572. [CrossRef]

16. Wang, C.; Zhu, J.; Wu, M.; Hou, J.; Zhou, H.; Meng, L.; Li, C.; Zhang, W. Multi-scale design and optimization for solid-lattice hybrid structures and their application to aerospace vehicle components. Chin. J. Aeronaut. 2020, in press. [CrossRef]

17. Vo-Duy, T.; Ho-Huu, V.; Do-Thi, T.; Dang-Trung, H.; Nguyen-Thoi, T. A global numerical approach for lightweight design optimization of laminated composite plates subjected to frequency constraints. Compos. Struct. 2017, 159, 646-655. [CrossRef]

18. Fan, H.-T.; Wang, H.; Chen, X.-H. An optimization method for composite structures with ply-drops. Compos. Struct. 2016, 136, 650-661. [CrossRef]

19. Liu, Q.; Paavola, J. Lightweight design of composite laminated structures with frequency constraint. Compos. Struct. 2016, 156, 356-360. [CrossRef]

20. Honda, S.; Igarashi, T.; Narita, Y. Multi-objective optimization of curvilinear fiber shapes for laminated composite plates by using NSGA-II. Compos. Part B Eng. 2013, 45, 1071-1078. [CrossRef]

21. Awad, Z.K.; Aravinthan, T.; Zhuge, Y.; Gonzalez, F. A review of optimization techniques used in the design of fibre composite structures for civil engineering applications. Mater. Des. 2012, 33, 534-544. [CrossRef]

22. Zhu, X.; He, R.; Lu, X.; Ling, X.; Zhu, L.; Liu, B. A optimization technique for the composite strut using genetic algorithms. Mater. Des. 2015, 65, 482-488. [CrossRef]

23. Ling, H.; Ying, L.; Bin, Y.; Yueqin, Z. Dynamic modeling and simulation of flexible beam finite rotation with ANCF method and FFR method. Mechanics 2018, 24, 715-724. [CrossRef]

Publisher's Note: MDPI stays neutral with regard to jurisdictional claims in published maps and institutional affiliations. 